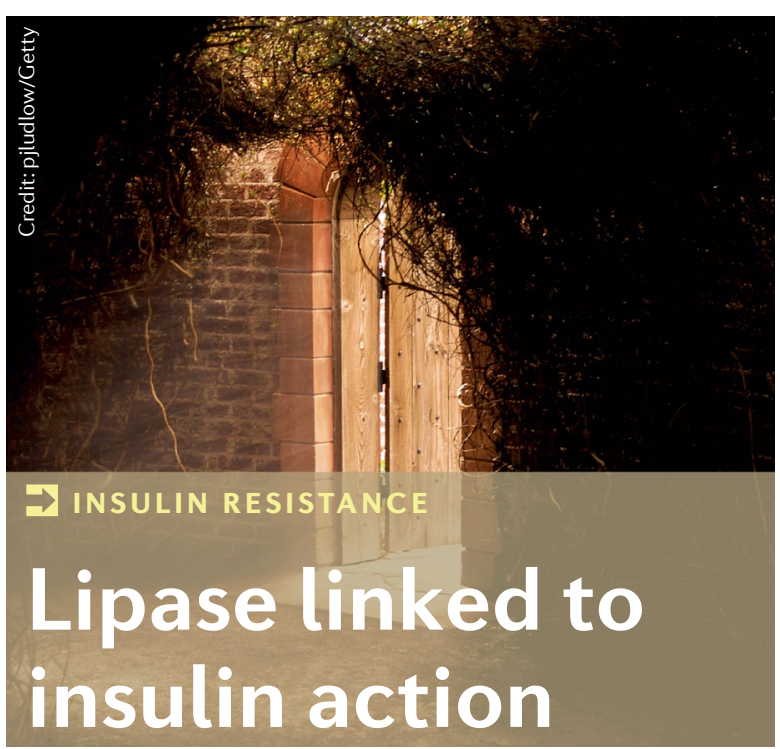

Impaired adipose tissue insulin signalling is a major feature of insulin resistance, but the precise mechanisms behind this impairment were unclear. Now, in a new study published in Nature Metabolism, Dominique Langin and colleagues have described a novel hormone-sensitive lipase-ChREBP interaction that constitutes a previously undescribed gateway between lipid and glucose metabolism.

"I started working on hormone-sensitive lipase in the 1990s during my post-doc at University of Lund, Sweden," adds Langin. "The initial period was devoted to cloning the human gene and characterization of various isoforms." Langin and his team have now described a new role for hormone-sensitive lipase, one that is independent of its enzymatic activities. In their study, Langin and colleagues describe a direct proteinprotein interaction between a lipase and the transcription factor ChREBP. The authors found that this interaction controls the intracellular localization and transcriptional activity of ChREBP.

To decipher the mechanisms involved in improvements in glucose metabolism mediated by hormone-sensitive lipase inhibition, the authors used human adipocytes and mouse transgenic models. They also used a number of techniques, including surface plasmon resonance assays, to investigate protein-protein interactions. Once Langin and colleagues had characterized this new pathway, they provided translational relevance through experiments on adipose tissue samples from different clinical studies.

"To combat obesity-associated insulin resistance and the development of type 2 diabetes mellitus, there is currently no strategy targeting adipose tissue (thiazolidinediones worked that way but were withdrawn several years ago for adverse effects unrelated to the control of fat metabolism)," explains Langin. "Disrupting the hormone-sensitive lipase-ChREBP interaction constitutes such a strategy that would be specific for adipose tissue as the lipase is only expressed at very low levels in the liver." Therefore, the authors are currently working towards improving the characterization of the domains of interaction in the two proteins with the aim of developing molecules to disrupt that interaction.

Alan Morris

ORIGINAL ARTICLE Morigny, P. et al. Interaction between hormone-sensitive lipase and ChREBP in fat cells controls insulin sensitivity. Nat. Metab. https://doi.org/10.1038/ s42255-018-0007-6 (2018)

\title{
Exploring the antidiabetic effects of FGF1
}

Intracerebroventricular injection of fibroblast growth factor 1 (FGF1) in a rat model of type 2 diabetes mellitus (T2DM) induces sustained remission of hyperglycaemia independently of insulin-dependent glucose clearance reports a new study in Diabetes.

"We previously reported that a single dose of the growth factor FGF1 delivered directly into the brain causes sustained remission of hyperglycaemia across multiple rodent models of T2DM," explains Michael Schwartz, corresponding author. "A key follow-up goal was to identify peripheral mechanisms mediating this effect."

The researchers used the Zucker diabetic fatty $f a / f a(Z D F)$ rat model of T2DM in their current study as it closely approximates human T2DM as both are characterized by progressive loss of $\beta$-cell function and mass over time.

In $\mathrm{ZDF}$ rats that received the intracerebroventricular injection of FGF1, the anticipated decline in $\beta$-cell function was delayed, corresponding to the time that hyperglycaemia was in remission. In addition, compared with control animals and baseline values, $\beta$-cell mass was increased in FGF1-treated animals 3 weeks after injection. However, after 7 weeks, $\beta$-cell mass decreased to match the values in control rats. Thus, FGF1 delays but does not prevent loss of $\beta$-cell function and diabetes mellitus relapse in ZDF rats.

Notably, a frequently sampled intravenous glucose tolerance test in a separate cohort of ZDF rats demonstrated that treatment with intracerebroventricular FGF1 had no effect on glucose-induced insulin secretion or insulin sensitivity. Furthermore, intravenous insulin injection had a reduced glucoselowering effect in rats that received FGF1 compared with control rats. The researchers concluded that the sustained remission of hyperglycaemia induced by FGF1 must be insulin independent.

Therefore, Schwartz and colleagues decided to focus on hepatic glucose uptake, which is insulin independent, as a possible mechanism contributing to remission of hyperglycaemia. They used a mathematical model to provide estimates of hepatic glucokinase activity (a key step in hepatic glucose uptake), which was reported to be doubled in ZDF rats that received FGF1. These estimates were in agreement with the twofold increases in hepatic Gck gene expression and GCK enzymatic activity measured biochemically in the livers of these rats. These data seem to confirm the researchers' hypothesis that hepatic glucose uptake has a role in mediating the effects of FGF1 in ZDF rats.

"Our key goals are to determine if changes in autonomic input to the liver and pancreas account for our findings," says Schwartz. "In addition, we are making progress identifying the brain area, cell types and signal transduction mechanisms that are responsible for this FGF1 effect."

Isobel Leake

ORIGINAL ARTICLE Scarlett, J. M. et al. Peripheral mechanisms mediating the sustained anti-diabetic action of FGF1 in the brain. Diabetes https://doi.org/10.2337/db18-0498 (2018)

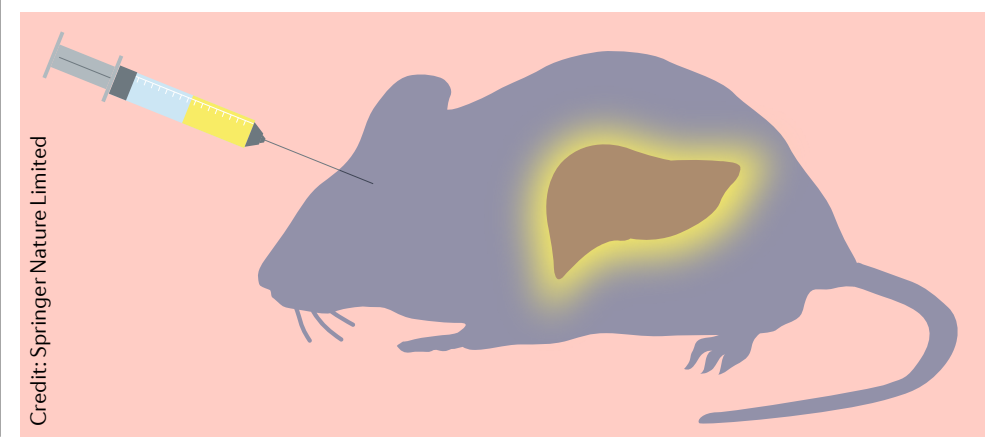

